\title{
ENSAYO
}

\section{¿Derecho a morir con dignidad?}

\author{
Álvaro Ruiz \\ Departamento de Medicina Interna, Facultad de Medicina, Pontificia Universidad Javeriana; \\ Departamento de Epidemiología Clínica y Bioestadística, Facultad de Medicina, \\ Pontificia Universidad Javeriana, Bogotá D.C., Colombia
}

\begin{abstract}
El derecho a morir con dignidad es un concepto vago y que recibe múltiples interpretaciones, muchas de ellas inapropiadas. Se propone la necesidad de que sea el médico quien se haga responsable de proteger los derechos del paciente, de garantizar el uso racional de los recursos y de velar porque las decisiones sean apropiadas y proporcionadas a la situación del paciente, a su pronóstico, expectativas y deseos, pero también, a la utilidad esperada y a la relación costo-beneficio. Se enfatiza que la dignidad puede ser entendida de muchas maneras y que, a veces, en su nombre se somete al paciente, o lo hace él mismo, a sufrimientos, dolor y complicaciones que podrían haberse evitado, no necesariamente en busca de prolongar la vida. Se hace énfasis en que, cuando no hay probabilidades razonables de supervivencia 0 cuando no puede ya buscarse mejorar la calidad de vida, deben enfocarse los esfuerzos en procurar calidad de muerte.
\end{abstract}

Palabras clave: derecho a morir/ética, bioética, derechos del paciente, negativa del paciente al tratamiento, cuidado terminal, tanatología

\section{Right to die with dignity?}

The right to die with dignity is an ill-defined concept, with multiple, often inappropriate, interpretations. The current proposition is that the physician take full responsibility for protecting the patient's rights, for ensuring a rational use of resources and for overseeing the decisionmaking process such that the information is adequate and the steps proportioned. This responsibility extends not only to the health status of the patient situation, to the patient's prognosis, and to his/her expectations and wishes, but also to the benefits foreseen and to the cost-benefit ratio. Emphasis is placed on two aspects of this relationship. First, dignity can be interpreted in many ways and sometimes, in the name of dignity, the patient is exposed (or exposes him/herself) to suffering, pain and complications that can be avoided. Second, when no reasonable probability of survival is present and a better "quality of life" is impossible, efforts are better redirected to offering a better quality of death.

Key words: right to die/ethics, bioethics, patient rights, treatment refusal, terminal care, thanatology

Los médicos hemos cometido un error a lo largo de los años al hablar y permitir a los pacientes y al público en general comentar sobre el derecho a morir con dignidad, sin aclarar el alcance de la expresión.

\section{Correspondencia:}

Álvaro Ruiz, Departamento de Epidemiología Clínica y Bioestadística, Hospital Universitario de San Ignacio, Carrera 7 No. 40-62, Bogotá D.C., Colombia.

Teléfonos: 320 8320, extensión 2799, y 232-2198; fax: 285 6981

aruiz@javeriana.edu.co

Recibido: 16/07/07; aceptado: 28/02/08
Es un error que seguimos alimentando sin cesar, que toleramos e incluso incentivamos. Nuestra responsabilidad, no sólo con nuestros pacientes y con la opinión general, sino con nuestros estudiantes, ha sido descuidada porque caímos en la trampa del "derecho a morir con dignidad", una expresión con múltiples significados y que no ha sido aclarada suficientemente.

¿Tiene una persona "derecho a morir con dignidad"? Por supuesto que sí (1). Nadie lo dudaría. La opción de querer, llegado el momento, cerrar nuestros ojos en el mejor ambiente posible, 
rodeados por nuestros seres queridos, en paz con nosotros y con el mundo, es ideal. No dudamos en aceptar que, en el momento de la muerte, quisiéramos que así fuera (2-6).

Hay, sin embargo, algunas preguntas que debemos hacernos. En las respuestas encontraremos, con seguridad, el error que hemos cometido.

¿Es el hogar el mejor sitio para morir en compañía de los seres queridos? La respuesta es clara. Depende. Si una persona tiene una enfermedad terminal conocida, en la que los síntomas y molestias pueden ser controlados y su final puede llegar de manera pacífica y tranquila, el lecho propio es el mejor sitio para morir, con las personas más allegadas cerca (1).

Si bien el proceso de la muerte es doloroso, en este caso el paciente deberá estar preparado y las particularidades del proceso mismo de la muerte estarán bajo control médico: sin dolor, con control apropiado de la ansiedad, sin asfixias extremas ni hemorragias incontrolables. Y también para la familia el médico debe haber preparado la situación, con una clara explicación de cómo la enfermedad no tiene solución y que ya llegó el momento de cambiar de marcha (1). Los esfuerzos no se dirigirán ya al intento de prolongación de la vida con calidad al que estaban enfocados (2). Se cambia a un nuevo objetivo: dada la irreversibilidad o la imposibilidad de recuperación del caso, toda la energía se enfocará a proporcionar al paciente la mejor "calidad de muerte" posible.

Se habrá preparado a la familia para que entienda la situación, para que cambie su sentimiento (en este momento, egoísta) de querer tener a su ser querido por más tiempo, por un sentimiento altruista de aceptar lo que es mejor para él en este momento. Y lo mejor, dada la imposibilidad de recuperarse, es morir. Y morir en las mejores condiciones posibles.

En este caso, la muerte llega sin aspavientos, a un ámbito que la espera con tristeza pero, al mismo tiempo, con la resignación de que se trata de una solución a un problema que tiene en sufrimiento a nuestro ser querido. Se logra, así, una muerte digna.
Pero no siempre se logra la conjunción de estos elementos. Con frecuencia se espera de un enfermo terminal una muerte incómoda y escandalosa, con hemorragia digestiva alta y la consecuente broncoaspiración y dificultad respiratoria progresiva; o una lenta pero dolorosa y muy dramática asfixia en los casos de enfermedades crónicas respiratorias; o la horrible hiperventilación central y movimientos de descerebración de la herniación cerebral (7).

Hay muchos ejemplos de muertes que producirán recuerdos imborrables en los familiares que asisten, impotentes, al proceso. Y no es infrecuente que, aún con conocimiento de causa, en el último momento decidan llevar a sus familiares en estado agónico al servicio de urgencias de un hospital. La muerte ocurre en medio de angustia, carreras, ambulancias que tardan en llegar, servicios de urgencias inhumanos o demorados que tardan en comprender la situación, explicaciones incoherentes de parte y parte ante la emergencia y, peor aún, con frecuencia en medio de inútiles, desproporcionadas y espeluznantes maniobras de resucitación $(5,7)$.

Si el médico prevé este posible final, ¿no es su obligación explicarlo al paciente y la familia? Debe prepararlos para lo que pueda ocurrir y ofrecer el mejor escenario posible que puede ser, incluso, una unidad de cuidados intensivos. ¿Para lograr salvar la vida cuando ocurra la hemorragia masiva o cuando sobrevenga la crisis de dificultad respiratoria irreversible? No. Claramente no, y es el deber del médico estar a cargo. Para controlar la situación y evitar al enfermo una muerte con dolor o grave ansiedad $(2,3)$. Para ayudar a la familia a entender el proceso, a sobrellevarlo con la tranquilidad de que es la mejor opción en el momento. Para apoyarlos en la aceptación de lo irreversible (4-6).

$Y$, también, función fundamental, para asegurarse de que el equipo de salud a cargo del paciente sabe claramente lo que está ocurriendo y entiende que hay una persona en el tránsito a la muerte, y que el equipo debe apoyar ese tránsito de la mejor manera: con dignidad para todos, sin recurrir a intervenciones injustificadas, sin el uso de medicación o tecnología desproporcionada y sin 
desgaste de recursos. El equipo de salud tiene el deber de permitir la muerte digna y, además, tiene el deber de custodiar esa muerte digna (3).

Quiere decir que debe estar preparado para analizar de manera completa (con precisión científica, con solidaridad humana, con balance apropiado de riesgos, costos y beneficios) la situación a la que se enfrenta y las opciones disponibles. Se habla de proporcionalidad de los recursos. Si bien un marcapasos externo, un balón de contrapulsación, una hemodiálisis de urgencia o una cirugía extrema pueden ser medidas racionales (proporcionadas) en algunos casos, en los que se esperan resultados positivos para la salud del paciente, un antibiótico puede ser absolutamente desproporcionado ante una infección que podría acelerar la muerte (esperada) de un enfermo con cáncer irrecuperable, metástasis y dolores intensos (5).

Y hemos llegado a uno de los más sagrados deberes de un médico: el de analizar cuidadosamente cada decisión que toma y decidir cuándo es proporcionado utilizar medicamentos, tecnología o intervenciones y cuándo no lo es (2). $Y$ si se trata de un paciente terminal, con una enfermedad no recuperable, sopesar cuidadosamente cada pequeña decisión porque en esas circunstancias su habitual misión de procurar calidad de vida (idealmente acompañada por prolongación de la vida) debe cambiar a una aún más delicada, la misión de procurar calidad de muerte. Es un deber ineludible (4).

Deber... del que no se habla. Deber que ha sido omitido por pacientes, médicos, medios de comunicación, revistas científicas y autores. ¿Tiene el paciente derecho a morir dignamente? Por supuesto que sí. Pero, ¿no es triste que todo el énfasis en la literatura científica y periodística esté centrado en las acciones que debe tomar el individuo para protegerse de la ciencia y de los médicos, llegado el caso?

El error mencionado, al permitir que se hable indiscriminadamente de un concepto de "derecho a morir dignamente" sin mayores especificaciones, se refiere expresamente a la falta de claridad en las implicaciones de la expresión y a las responsabilidades de los autores.
Debe establecerse que es una obligación primaria e ineludible del médico y de su equipo terapéutico la vigilancia de que ese derecho a morir dignamente se respete y que las decisiones médicas busquen, activamente, esa dignidad y la proporcionalidad en las acciones.

Que el paciente y su familia tengan la tranquilidad y la certeza de que sus derechos a la vida, a la autonomía y a la dignidad estarán protegidos con celo y fervor por su médico. Y que ni él ni ellos tengan que buscar protegerse de aquéllos a quienes confían su salud y sus derechos, a la vida con calidad y a la muerte, oportuna y con dignidad, cuando llegue el momento.

¿No debería enfatizarse aún más o, al menos, por igual, el deber del médico de proteger la muerte digna de sus pacientes? No sólo de ofrecer todo su conocimiento, su capacidad, sus sentimientos y su sensibilidad. Esto es un deber elemental. Debe cumplirlo a cabalidad. Pero debe ir más allá: es su deber no sólo hacer cuanto esté a su alcance para dar dignidad a la muerte inevitable; también es su deber vigilar que así se haga, que nadie se sobrepase, que no se usen medidas desproporcionadas con el pronóstico el paciente, ni por parte de las instituciones, ni por parte de otras personas del equipo de salud.

Y también es su deber, su obligación ineludible, no dejarse presionar por peticiones de los familiares o allegados que en su desconocimiento de la situación, o en los sentimientos de culpa frecuentes en estos casos, o en su mal entendida solidaridad quieren que se prolongue la vida de quien ha llegado al fin de su vida y merece el tránsito en paz.

También es su deber evaluar cuidadosamente el concepto de dignidad, vago e impreciso que, con frecuencia, se esgrime equivocadamente para evitar una intervención que ofrecería dignidad a la muerte, o para rechazar una hospitalización, una cirugía, un procedimiento o un medicamento (4). Al hacerlo se somete al paciente (o se somete él mismo) a dolor o a franco sufrimiento, que podrían haber sido aliviados, no con la intención de salvar la vida sino de garantizar esa dignidad en nombre de la cual, de manera inapropiada, se rechazó en primer lugar la intervención. 
Nos hemos equivocado en la educación médica al no insistir en que una de nuestras obligaciones es la de utilizar racionalmente los recursos, tanto en quienes tienen esperanzas de mejorar como en quienes no la tienen. Debemos hacer énfasis en que es nuestra obligación sopesar cuidadosamente, siempre, los riesgos y costos de cualquier decisión que tomemos contra los beneficios posibles. Y es nuestra obligación evaluar siempre la proporcionalidad de lo que ofrecemos en relación con los potenciales resultados (6).

Si el resultado muestra que el balance se inclina por el beneficio, debemos empeñar todo nuestro esfuerzo, no sólo por aplicar la medicación o la intervención, sino por hacer conocer a nuestro paciente y a su familia el resultado del análisis hecho. Ellos tienen derecho a conocer el proceso, explicado de manera clara y concisa, en su lenguaje y con la verdad.

Pero, si el análisis muestra desproporcionalidad en el balance, también tenemos en ese momento tres deberes: evitar su uso, para lo cual evitaremos tomar la decisión que se demostró inapropiada; explicar al paciente y a sus allegados las razones por las cuales no hay justificación para las intervenciones, y finalmente, no menos importante, vigilar que se respete ese análisis. Quiere decir que tendremos que también hacer explícita la decisión al equipo de salud. Y dejar constancia para que, aun en el caso de que no estemos presentes, o que el paciente vaya a ser llevado a otra institución o vaya a quedar a cargo de otro equipo, haya claridad en las decisiones que se deban tomar (8).

¿Tiene el paciente derecho a morir con dignidad? Por supuesto que sí. Pero, por favor, complementemos el concepto. El médico tiene el deber de promoverla, de protegerla y hacerla respetar. $Y$ con enorme frecuencia, dado su conocimiento del paciente, de su familia y de su historial clínico, es el médico quien tiene la información completa que permitirá llegar a la decisión de cambiar de rumbo y dejar la búsqueda, ya mencionada, de la calidad de vida (idealmente con prolongación de la misma), para enfocarse en una misión tanto o más difícil, pero probablemente más humanitaria: el proceso de la calidad de muerte. La muerte con dignidad, que todos quisiéramos, que todos los médicos tenemos el deber de proteger.

\section{Conflictos de intereses}

Declaro expresamente que no tengo ningún conflicto de interés para el presente artículo.

\section{Financiación}

No se recibió financiación para la escritura de este ensayo.

\section{Referencias}

1. Swarte NB, van der Lee ML, van der Bom JG, van den Bout J, Heintz PM. Effects of euthanasia on the bereaved family and friends: a cross sectional study. BMJ. 2003;327:189.

2. Grayling AC. Right to die. BMJ. 2005;330:799.

3. Hardwig J. Is there a duty to die? Hastings Cent Rep. 1997;27:34-42.

4. Chochinov HM, Hack T, McClement S, Kristjanson L, Harlos M. Dignity in the terminally ill: a developing empirical model. Soc Sci Med. 2002;54:433-43.

5. Turner K, Chye R, Aggarwal G, Philip J, Skeels A, Lickiss JN. Dignity in dying: a preliminary study of patients in the last three days of life. J Palliat Care. 1996;12:7-13.

6. Mendoza-Vega J. El derecho a morir con dignidad. Biomédica. 2005;25:165-6.

7. Macklin R. Dignity is a useless concept. BMJ. 2003;327:1419-20.

8. Ashcroft RE. Making sense of dignity. J Med Ethics. 2005;31:679-82. 